\title{
Multi-Screen Cyber-Physical Video Game: An Integration with Body-Area Inertial Sensor Networks
}

\author{
Chin-Hao Wu, Yuan-Tse Chang, and Yu-Chee Tseng \\ Department of Computer Science, National Chiao Tung University \\ Hsin-Chu, 30010, Taiwan
}

\begin{abstract}
Deploying body-area inertial sensor networks on human bodies to capture motions has attracted a lot of interests recently, especially in cyber-physical video games and contextaware applications. While video games on the cyber world have been quite popular, enhancing them with more physical inputs, such as those from inertial sensors, is becoming a new trend. Following this trend, we develop a video game integrated with body-area inertial sensor networks deployed on players as inputs and with multiple game screens to broaden players' views and provide more realistic interaction experiences. Our design simulates a multi-screen game engine by controlling a set of game engines simultaneously. A prototype with a body-area inertial sensor network platform, a cyber-physical game controller, and a set of game engines is demonstrated. The demonstrated game also addresses the interaction between virtual objects and players.
\end{abstract}

\section{INTRODUCTION}

Cyber-physical systems (CPS) have drawn a lot of attention recently. In the past two decades, cyber systems were one of the fastest growing areas due to the advance of mobile computing technologies (such as portable computers and smart phones) and the standardization of various wireless communication and networking technologies (such as ZigBee, WiFi, Bluetooth, WiMAX, 2G/3G/3.5G, LTE, etc.), which are tightly integrated under the Internet environment. On the other hand, a major advance in physical systems is the development of Micro Electro Mechanical Systems (MEMS), which can greatly enrich cyber systems with more physical inputs and actuators.

We are interested in CPS for cyber-physical video games. Traditionally, cyber video games are supported by game engines that take players' inputs from keyboards and joysticks. Enhancing such systems with more physical inputs, such as body motions captured by inertial sensors, has attracted a lot of attention recently. We call such systems cyber-physical games. The Nintendo Wii [1] is one example with MEMS-based inertial sensors. Reference [2] shows that by using MEMSbased inertial sensors and a wired network, body motions can be reconstructed through computer animation with little distortion. Wireless-based body-area inertial sensor networks (BISNs) have been studied in [3]-[6]. On the other hand,

Y.-C. Tseng's research is co-sponsored by MoE ATU Plan, by NSC grants 96-2218-E-009-004, 97-3114-E-009-001, 97-2221-E-009-142-MY3, and 982219-E-009-005, by MOEA 98-EC-17-A-02-S2-0048, and 98-EC-17-A-19S2-0052, and by ITRI, Taiwan.

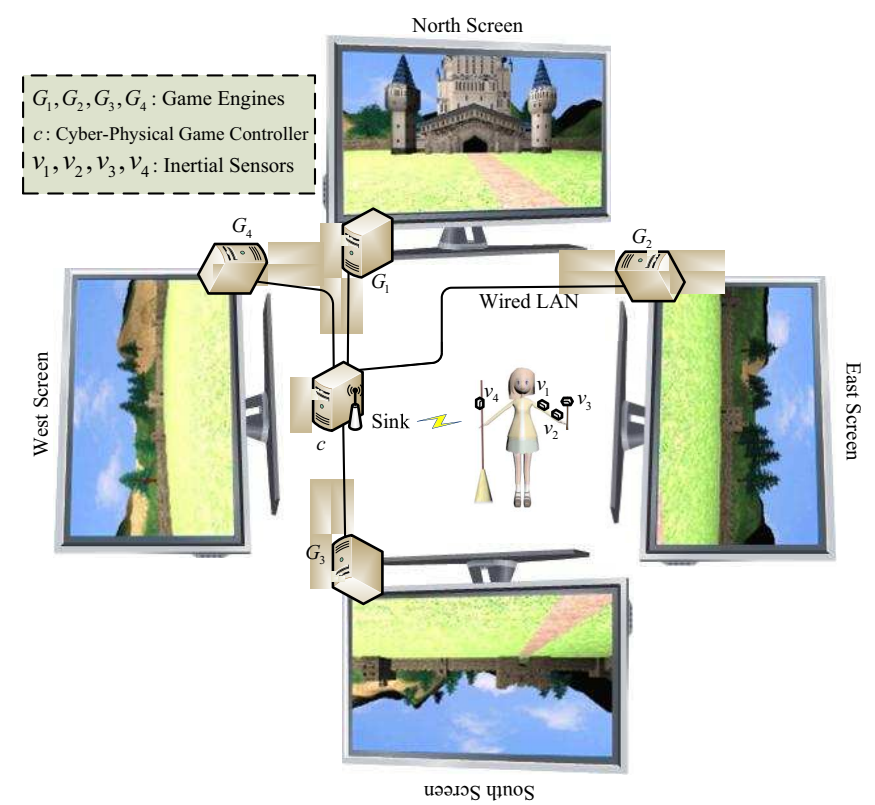

Fig. 1. System architecture.

new display technologies, such as LCD, plasma, and backprojection displays, can further enrich cyber-physical games with better visual effects [7], [8].

The above advances motivate us to develop a multi-screen cyber-physical video game with physical inputs from players equipped with BISNs. We demonstrate our prototype of the BISN platform, the Cyber-Physical Game Controller, and the Game Engines.

\section{SyStem DESCRIPTION}

Our system architecture is shown in Fig. 1. We consider a single-player game. A BISN is deployed on the player. The sensing data is collected by the sink node attached to the Cyber-Physical Game Controller, which will process the sensing data and feed it to four game engines $G_{1}, G_{2}, G_{3}$, and $G_{4}$ via wired LAN interfaces. The four game engines are set to four camera angles (east, west, north, and south) and they together provide a $360^{\circ}$ panorama view of the game scene. The software architecture is shown in Fig. 2. Below, we describe 


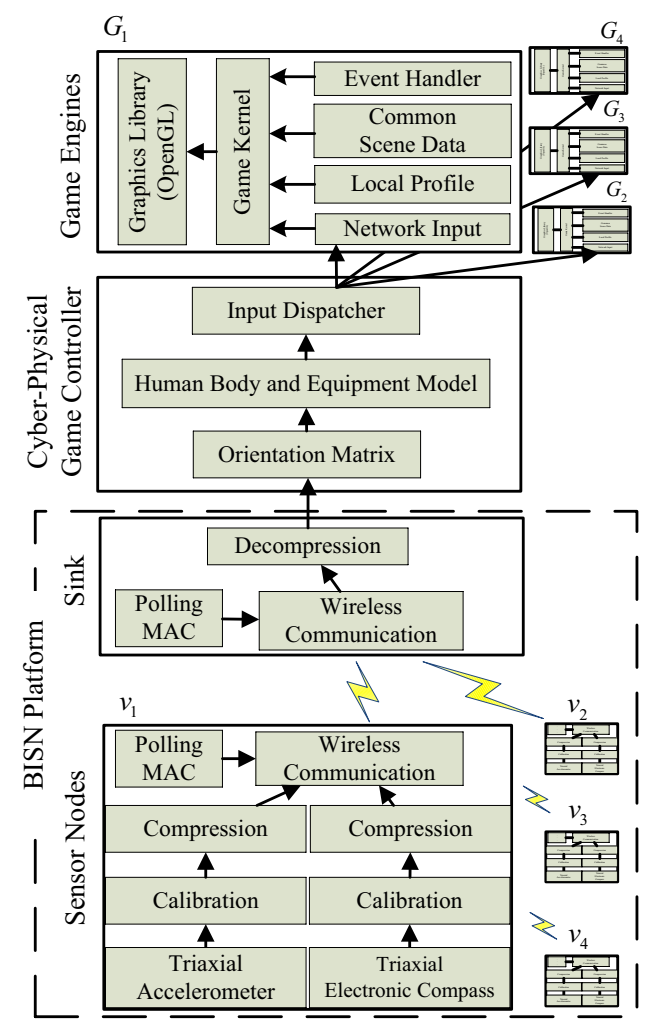

Fig. 2. Software architecture.

each component.

BISN Platform: The platform consists of one sink node and four inertial sensor nodes $v_{1}, v_{2}, v_{3}$, and $v_{4}$. Each node consists of some inertial sensors, a micro-controller, and a wireless module. In our current implementation, each node is equipped with a triaxial accelerometer and a triaxial electronic compass. The triaxial accelerometer senses the gravity in $\mathrm{x}, \mathrm{y}$, and $\mathrm{z}$ axes, while the triaxial compass senses the earth magnetic force in $\mathrm{x}, \mathrm{y}$, and z-axes. The "Calibration" module removes noises and converts sensing signal into meaningful data. Since sensing data is streaming data, the "Compression" module executes a differential coding to reduce data transmission. The compressed data is reported to the sink through the "Wireless Communication" module. The sink node runs a "Polling MAC" to avoid collisions and to improve throughput.

Cyber-Physical Game Controller: This component has two main functions: 1) to convert sensing data into game inputs (by "Orientation Matrix" and "Human Body and Equipment Model") and 2) to dispatch game inputs to each game engine (by "Input Dispatcher"). Fig. 3 illustrates the conversion process. The player wears four inertial sensor nodes, one on the broomstick, one on forearm, one on upper arm, and one on the club. From the sensing inputs, the "Orientation Matrix" represents the absolute orientations of all sensor nodes with respect to the earth coordinate. The "Human Body and Equipment Model" derives the direction of the broomstick (by its $\hat{x}$ axis) and two articulation angles $\theta_{1}$ and $\theta_{2}$ (by orientation matrices and limb lengths). These parameters are then fed to the game engines via wired LAN interfaces. Typical game engines do not support multiple screens. Our game controller tries to simulate a multi-screen game engine by sending proper data, via the "Input Dispatcher" to four game engines, as explained below.

Game Engines: Each game engine has a "Game Kernel", which takes inputs from four modules. The "Common Scene Data" module describes the virtual world. It is the same for all game engines. The "Network Input" module receives game inputs from the "Cyber-Physical Game Controller" and updates the virtual world in the game. The "Event Handler" module processes the interactions, especially collisions, between virtual objects and the player. For example, when the player hits a ball, it bounces away, and the "Event Handler" increases the player's score. Each game engine contains a camera, whose direction is specified by the "Local Profile", that takes pictures for its virtual world and feeds the captured data to the "Game Kernel", which calls the "Graphics Library" to display the results. Since we have four game engines with four cameras facing to east, west, north, and south, a $360^{\circ}$ panorama view of the virtual world is provided to support better visual effect to the player.

\section{Demonstration Scenario}

We adopt the Quidditch sports in "Harry Potter" [9] as our game scenario. The player rides a flying broom in a practice field, and she tries to attack approaching balls by her club. She flies at a constant speed and controls her broomstick to change directions.

For the BISN platform, we adopt Jennic JN5139 [10] and OS5000 sensor [11] (refer to Fig. 3). Each Jennic JN5139 consists of a micro-controller and a IEEE 802.15.4 wireless transmission module, and each OS5000 has a triaxial accelerometer and a triaxial electronic compass. The sampling rate is set to $20 \mathrm{~Hz}$, a common value for motion capturing [12]. The average packet loss rate of our polling MAC is about $0.16 \%$, and the average delay for collecting a complete set of sensing data from a node is around $83 \mathrm{~ms}$.

Fig. 4 shows some snapshots of the game. The player flies a short distance to the north in Fig. 4(a), and then she turns to the west in Fig. 4(b). We adopt Unity [13] as our game engine, which features a fully integrated editor and a physics engine for rapid 3D game prototyping. Fig. 5(a) and Fig. 5(b) show the corresponding settings of the west and the north game engines in Fig. 4(a), respectively. The virtual character, who is controlled by the player, looks to the north in both game engines. The camera of Fig. 5(a), whose viewing volume is illustrated by white lines, looks to the west, while the camera of Fig. 5(b) looks to the north. Fig. 5(c) and Fig. 5(d) correspond to the west and the north screens in Fig. 4(b), respectively, where both of the player and the virtual character look to the west, but the directions of the cameras remain unchanged.

\section{REFERENCES}

[1] Nintendo, "Wii," http://wii.com. 


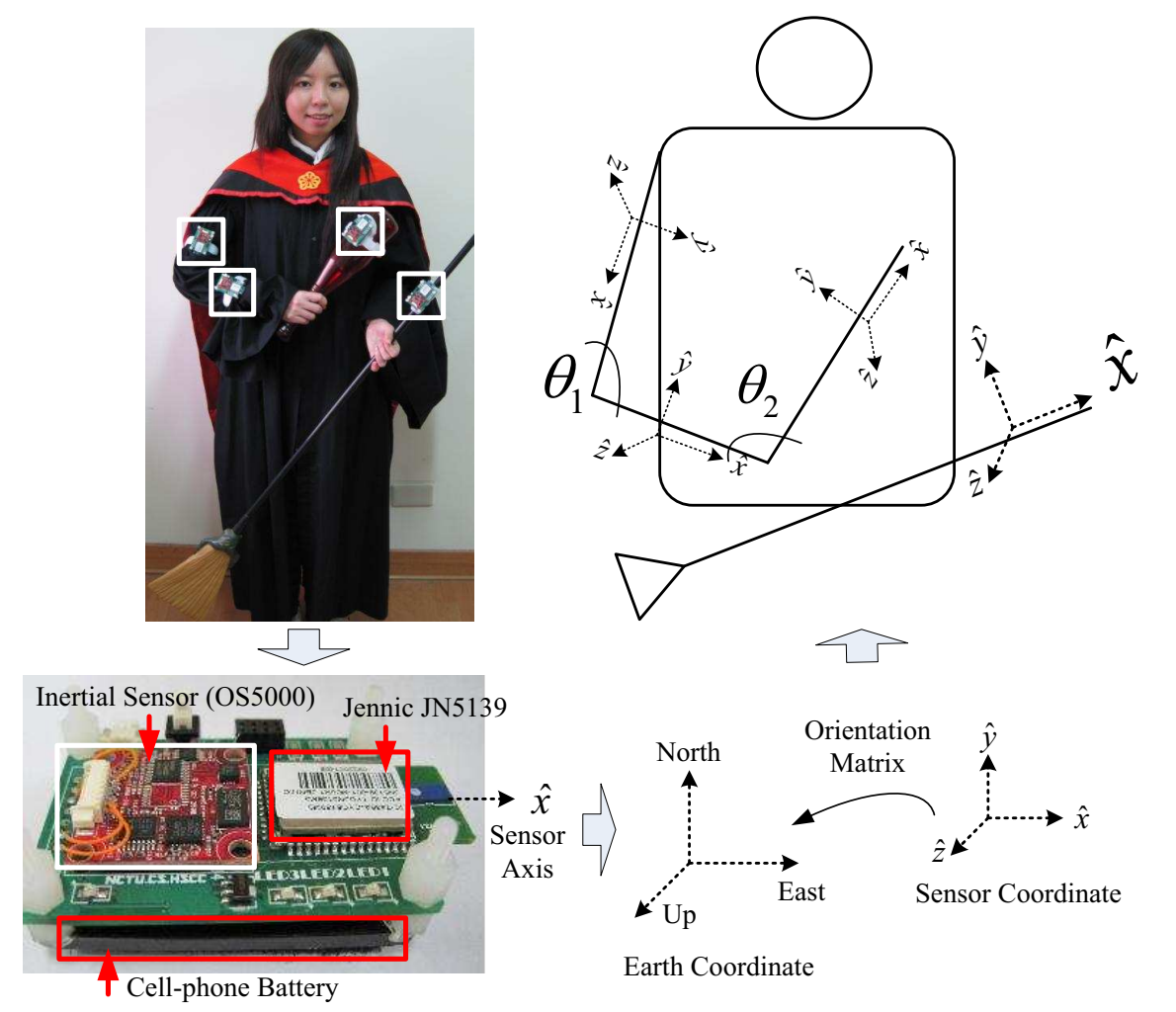

Fig. 3. Human body and equipment model.

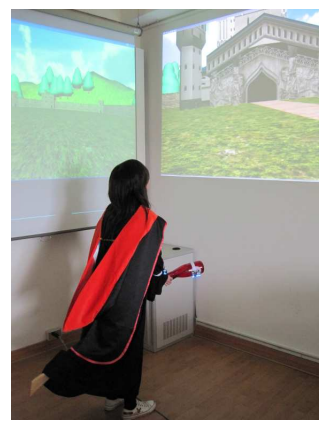

(a)

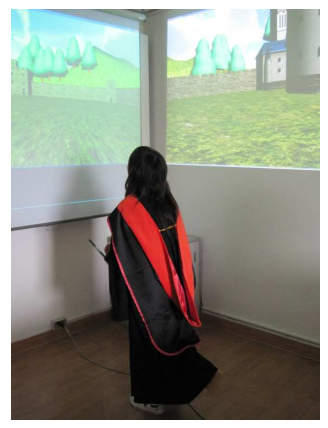

(b)

Fig. 4. Snapshots of the game.

[2] D. Vlasic, R. Adelsberger, G. Vannucci, J. Barnwell, M. Gross, W. Matusik, and J. Popović, "Practical motion capture in everyday surroundings," ACM Trans. Graph., vol. 26, no. 3, p. 35, 2007.

[3] G. Zhou, J. Lu, C.-Y. Wan, M. Yarvis, and J. Stankovic, "BodyQoS: Adaptive and radio-agnostic QoS for body sensor networks," in Proc. of IEEE INFOCOM, 2008.

[4] D. Jea, W. Wu, W. J. Kaiser, and M. B. Srivastava, "Approximate data collection using resolution control based on context," in Proc. of Int'l Workshop on Wearable and Implantable Body Sensor Networks (BSN), 2007.

[5] L. Cheng, S. Hailes, Z. Cheng, F.-Y. Fan, D. Hang, and Y. Yang, "Compressing inertial motion data in wireless sensing systems - an initial experiment," in Proc. of Int'l Workshop on Wearable and Implantable Body Sensor Networks (BSN), 2008.

[6] P. K. Baheti and H. Garudadri, "An ultra low power pulse oximeter sensor based on compressed sensing," in Proc. of Int'l Workshop on Wearable and Implantable Body Sensor Networks (BSN), 2009.

[7] Panasonic, "Life Wall - gesture-controlled interactive TV," http://www.panasonic.com/cesshow, 2008.
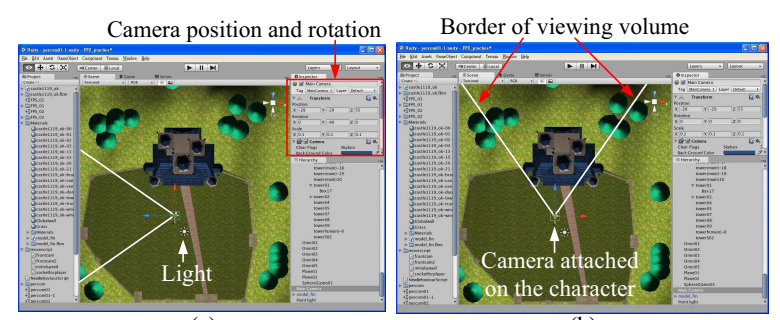

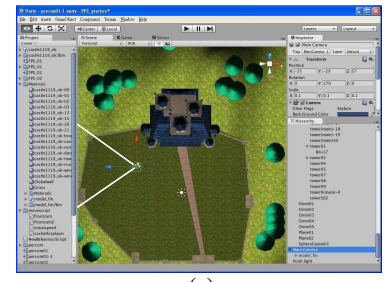

(c)

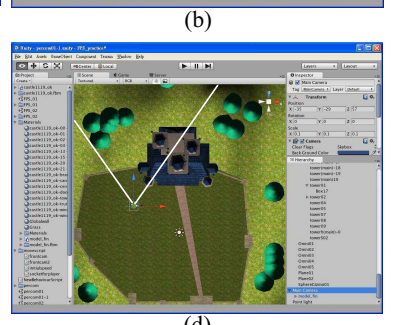

(d)
Fig. 5. Virtual character and camera settings in Fig. 4.

[8] H. Kim and D. W. Fellner, "Interaction with hand gesture for a backprojection wall," in Computer Graphics International Conference, 2004.

[9] J. K. Rowling, "Quidditch," http://en.wikipedia.org/wiki/Quidditch.

[10] Jennic, "JN5139," http://www.jennic.com.

[11] OceanServer, "OS5000 Family - Triaxial Accelerometer," http://www.ocean-server.com, 2008.

[12] C. V. C. Bouten, K. T. M. Koekkoek, M. Verduin, R. Kodde, and J. D. Janssen, "A triaxial accelerometer and portable data processing unit for the assessment of daily physical activity," IEEE Trans. Biomedical Engineering, vol. 44, no. 3, pp. 136-147, 1997.

[13] Unity, "Unity 2.5 - 3D Game Engine," http://unity3d.com, 2009. 